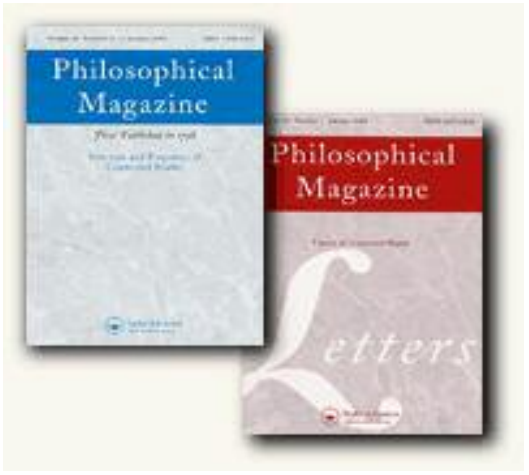

\title{
Evidence from numerical modelling for 3D spreading of [001] screw dislocations in Mg2SiO4 forsterite
}

\begin{tabular}{|r|l|}
\hline Journal: & Philosophical Magazine \& Philosophical Magazine Letters \\
\hline Manuscript ID: & TPHM-08-Jun-0229.R1 \\
\hline Journal Selection: & Philosophical Magazine \\
\hline Author: & 21-Jul-2008 \\
\hline Complete List of Authors: & $\begin{array}{l}\text { Carrez, Ph; Université des Sciences et Technologies de Lille, } \\
\text { Laboratoire de Structure et Propriétés de I Etat Solide } \\
\text { Walker, Andrew; University of Cambridge, Department of Earth } \\
\text { Sciences } \\
\text { Metsue, Arnaud; Université des Sciences et Technologies de Lille, } \\
\text { Laboratoire de Structure et Propriétés de I Etat Solide } \\
\text { Cordier, P; Université des Sciences et Technologies de Lille, } \\
\text { Laboratoire de Structure et Propriétés de I Etat Solide }\end{array}$ \\
\hline Keywords: & computer modelling, defect structures, dislocations, minerals \\
\hline Keywords (user supplied): & computer modelling, defect structures, dislocations \\
\hline & \multicolumn{2}{|l}{} \\
\hline
\end{tabular}

\section{๑) ScholarONE" \\ Manuscript Central}




\title{
Evidence from numerical modelling for 3D spreading of [001] screw dislocations in $\mathrm{Mg}_{2} \mathrm{SiO}_{4}$ forsterite
}

\author{
Ph. Carrez ${ }^{\mathrm{a}}$, A.M. Walker ${ }^{\mathrm{b}}$, A. Metsue ${ }^{\mathrm{a}}$ and P. Cordier ${ }^{\mathrm{a}}$ \\ ${ }^{a}$ Laboratoire de Structure et Propriétés de l'Etat Solide, UMR CNRS 8008, Université des \\ Sciences et Technologies de Lille, 59655 Villeneuve d'Ascq Cedex, France \\ ${ }^{b}$ Department of Earth Sciences, University of Cambridge, Downing Street, Cambridge, CB2 3EQ, \\ $U K$ \\ Second version, revised July, 20 $0^{\text {th }}, 2008$
}

Corresponding author:

Patrick Cordier

Laboratoire de Structure et Propriétés de l'Etat Solide - UMR CNRS 8008

Université des Sciences et Technologies de Lille - Bat C6

59655 Villeneuve d'Ascq Cedex - FRANCE

Phone: (33) 0320434341

Fax: (33) 0320436591

E-mail: Patrick.Cordier@univ-lille1.fr

Manuscript prepared with Word X on Macintosh 


\begin{abstract}
Computer simulations have previously been used to derive the atomic scale properties of the cores of screw dislocations in $\mathrm{Mg}_{2} \mathrm{SiO}_{4}$ forsterite by direct calculation using parameterised potentials and via the Peierls-Nabarro model using density functional theory. We show that, for the [001] screw dislocation, the parameterised potentials reproduce key features of generalised stacking fault energies when compared to the density functional theory results, but that the predicted structure of the dislocation core differs between direct simulation and the Peierls-Nabarro model. The [001] screw dislocation is shown to exhibit a low-energy non-planar core. It is suggested that for this dislocation to move its core may need to change structure and form a high-energy planar structure similar to that derived from the Peierls-Nabarro model. This could lead to dislocation motion via an unlocking-locking mechanism and explain the common experimental observation of long straight screw dislocation segments in deformed olivine.
\end{abstract}

Keywords: dislocation core, forsterite, numerical modelling, screw dislocations 


\section{Introduction}

As the dominant constituent of the Earth's upper mantle, the rheology of $(\mathrm{Mg}, \mathrm{Fe})_{2} \mathrm{SiO}_{4}$ olivine plays a controlling role in solid-state mantle convection and its surface expression, plate tectonics. Numerous experimental studies have been undertaken to identify active slip systems in deformed olivine and the magnesium end-member, forsterite [1-3]. In olivine, the Burgers vectors correspond to the shortest lattice repeats of the orthorhombic (space group Pbnm) crystal structure, [100] and [001]. At ambient pressure, under conditions of lowtemperature and high-stress, [001] dislocations gliding on (100), \{110\} and (010) are usually reported whereas [100] dislocations gliding on (010), (001), \{031\}, \{021\} and $\{011\}$ dominate at high temperature [4]. No other slip systems are observed despite the fact that, according to the von Mises criterion [5], there are not enough active slip systems to permit polycrystalline deformation. Recent studies have shown that [001] glide is enhanced when pressure increases [6-10] or when trace amounts of water are incorporated into the olivine crystal structure $[11,12]$.

In a recent study [8] Durinck et al. have derived dislocation core structures based on the Peierls-Nabarro (PN) model. The tendency of [001] screw dislocations to spread into (100) and $\{110\}$ glide planes led Durinck et al. [8] to speculate that [001] screw dislocations in olivine may exhibit a non-planar core, similar to that found in body centred cubic (bcc) metals (e.g. [13-15]). This hypothesis is supported by microstructural observations which always show that [001] glide is dominated by long straight screw dislocations (for instance $[16,2,6])$, implying that these dislocations experience large lattice friction stresses.

In the following, we want to clarify this assumption using results of full atomistic calculations of the [001] screw dislocation in forsterite. We compare the core structure of the [001] screw dislocation in forsterite calculated via direct atomistic simulation with that predicted by the PN model. For the direct atomistic simulations we reuse the results of calculations previously described by Walker et al. $[17,18]$. For consistency we calculate the generalised stacking fault (GSF) energies using the same potential model as that used for the direct simulations before applying the PN model to extract the core structure. The GSF calculations closely follow the methodology used by Durinck et al. [7, 8], who utilised density functional theory (DFT). This provides the opportunity to compare the GSF results of the potential model with those of DFT, allowing us to assess the ability of the potential model to cope with non-elastic deformation found at the core of dislocations.

\section{Methodology}


As recently reviewed by Schoeck [19, 20] and Woodward [21], the arrangement of atoms around a dislocation line can be obtained in two ways, either directly, using atomistic calculations, or from the Peierls-Nabarro model. Because of the computationally demanding need to include a large number of atoms, usually too many for ab initio methods, direct calculations are generally limited by the accuracy of a chosen interatomic potential model. This can be an important limitation [20], but fully ab initio simulations of dislocation cores in some simple materials are now becoming possible [22]. On the other hand, this approach requires few assumptions: in the simplest case the system must be periodic along the dislocation line and some approximate initial dislocation geometry must be used. The assumptions of the PN model are more severe. When one-dimensional GSF energies are used as input only pure edge or pure screw dislocations can be considered and spreading of the core is limited to the chosen glide plane [19]. However, the PN model can yield results in close agreement with direct atomistic calculations, even when the core structure is complex and the dislocation is dissociated, as is the case of $\mathrm{NiAl}[23,24]$.

\subsection{Direct atomistic simulation}

The direct atomistic simulations are described in detail elsewhere [17, 18], but key features of the methodology will be outlined here. The simulations were performed using a simulation cell that is periodic along the dislocation line and exhibits no periodicity in the two perpendicular directions. Strain associated with the dislocation was assumed to be well represented by anisotropic linear elasticity far from the core but this breaks down when the strain becomes large close to the core. In order to handle the non-linear effects and the atomic scale reconstruction of the core an atomistic model was used and this was embedded in the long-ranged elastic representation of the dislocated crystal. Although the elastic region can be allowed to respond to forces generated by the dislocation core (e.g. [21]) in these calculations the atoms around the rim of the simulation cell were held fixed at the location predicted by linear elasticity. The location of these atoms was therefore determined from only the dislocation's Burgers vector and elastic constants appropriate to the atomic model.

For the model of forsterite, an interatomic potential model with formal charges, fitted to the structure and some properties of quartz and binary oxides, was selected [25-27]. This model has been extensively used for studies of the thermodynamic and structural properties of perfect forsterite [28-30] as well as studies of surfaces [31-33] and point defects [34-37]. Parameters of the model are given in Table 1. The use of a potential model with a long-ranged Coulomb component implies the need for special treatment of this part of the energy 
calculation [17] and for care in building the simulation cell to avoid the creation of a charged simulation cell, or a cell with a large electrostatic dipole [18]. For the case of the [001] screw dislocation in forsterite this was achieved by using the Coulomb summation of Saunders et al. [38] and by building the simulation cell out of charge neutral units. The simulation cell has a radius of $75 \AA$ and atoms in the outermost $25 \AA$ rim were held fixed at the location given by linear elasticity. The model contained 15044 particles made up of 2658 magnesium atoms, 1330 silicon atoms, 5528 oxygen cores and 5528 oxygen shells. The initial location of all the atoms were determined from linear elasticity and the inner atoms are moved to minimise the total energy using the GULP code [39].

\subsection{Simulation via the Peierls-Nabarro model}

The alternative method used to arrive at a geometry of the dislocation core involves solving the Peierls-Nabarro equation, which relates a continuous distribution of shear in the glide plane, $S(x)$, to a continuous distribution of infinitesimal dislocations with density $\rho(x)$ and a restoring force, $F(S(x))$ :

$$
\frac{K}{2 \pi} \int_{-\infty}^{+\infty} \frac{1}{x-x^{\prime}}\left[\frac{d S\left(x^{\prime}\right)}{d x^{\prime}}\right] d x^{\prime}=\frac{K}{2 \pi} \int_{-\infty}^{+\infty} \frac{\rho\left(x^{\prime}\right)}{x-x^{\prime}} d x^{\prime}=F(S(x))
$$

where $K$ is the energy coefficient and $x$ and $x$ ' are displacements in the glide plane. For any amount of shear the restoring force can be calculated from a set of atomistic models containing generalised stacking faults with different displacements. The restoring force is the gradient of the GSF energy with respect to displacement and full details of the solution are given in [8], which follows the methodology of Joos et al. [40]. GSF calculations were performed with the same potential model and computer code as was used for the direct modelling of the core. The geometry of the supercells used for these GSF calculations were chosen to be the same as those used for the DFT based GSF calculations [7] and identical relaxation conditions have been used (i.e. atoms were only allowed to move in a direction perpendicular to the stacking fault during the calculation). Using the same simulation cell as the previous DFT calculations allows the use of the DFT results as a reference: it is possible to directly compare the DFT and parameterised potential GSF calculations and understand the limitations of the potential model with respect to dislocation modelling.

Solving the PN equation provides the shear profile, $S$, and the distribution of Burgers vector density, $\rho$, across the dislocation core. In order to display the atomic arrangement in the dislocation core, we follow the approach for screw dislocations described by Eshelby [41] and reproduced in [42]. Thus, we start from a perfect crystal structure split into two semi-infinite 
half crystals with a disregistry of $b / 2$ for symmetry reasons. The atom positions in the (001) plane (normal to the dislocation line) are given as $(x, y)$. The PN dislocation is then introduced by attributing to all atoms a displacement, $u$, along the dislocation (parallel to the $z$ coordinates axis) calculated from the disregistry function $S(x)$. Finally, the core structure is given by introducing the elastic displacement field $u_{z}(x, y)$. For a [001] screw dislocation calculated in this study with anisotropic elasticity the displacement field is found from:

$$
u_{z}(x, y)=\frac{1}{2 \pi} \int_{-\infty}^{+\infty} \rho\left(x^{\prime}\right) \arctan \left(\sqrt{\frac{S_{44}}{S_{55}}} \frac{y}{x-x^{\prime}}\right) d x^{\prime}
$$

where $S_{44}$ and $S_{55}$ are elements of the reduced elastic compliance matrix [43, 44].

\subsection{Geometrical analysis of the core structure of the [001] screw dislocation}

Although both methods of simulating the dislocation core reveal a detailed atomic structure, any comparison between the two is difficult. This is mostly because the displacements are predominantly parallel to the dislocation line and at first glance, looking along the line, it is hard even to differentiate between the dislocated and perfect crystal. A powerful method of analysing the core structure of a screw dislocation is to superimpose a differential displacement map on top of the atomic structure of the core. In particular, the difference in total displacement of two adjacent atoms along the dislocation line can be plotted as an arrow between the two atoms. The arrow length is proportional to the difference in displacement. This is known as the Vitek representation [45]. In this representation a screw dislocation in an isotropic elastic body would be represented by a circle of arrows of equal length around the core, with the length decreasing logarithmically with distance from the core. For $<111>$ screw dislocations in bcc metals three equivalent directions are clearly seen, representing the three-fold spreading of the core in $\{110\}$ planes.

For forsterite, it is useful to only represent the differential displacement for the oxygen sublattice. Indeed, the oxygen sublattice of forsterite can be viewed as a slightly distorted hexagonal close packed (HPC) lattice and this leads to the maximum symmetry properties.

\section{Results}

Results of the GSF calculations for the three slip systems are presented in Figure 1 and key parameters of the dislocation calculated from the PN model are presented in Table 2; in both cases the previous DFT results are compared with results form the potential model. The 
shapes of the GSF curves are clearly similar for the parameterised potentials and first principles calculations. In particular, we found a single peak in the GSF curve for [001](010), whereas $[001](100)$ and $[001]\{110\}$ are characterized by a camel-hump double peak shape. Quantitatively, energies differ between the two methods, especially for the (100) and $\{110\}$ planes, with the maxima being larger for the potential model than for DFT. This excess in barrier height is likely to be due to the use of a potential model utilising formal charges, a similar effect was observed in a study of oxygen diffusion using the same potential model [46]. However, the ratio between the stacking fault energy (SFE, corresponding to the minimum exhibited at half shear) and the maximum of the GSF curves $\left(\gamma^{\max }\right)$ are more consistent and only differ for the (100) plane (Table 2). In agreement with [7], the SFE in the (100) and $\{110\}$ planes are found to be very similar with a value of $42 \mathrm{meV} / \AA^{2}$. Finally, for the (010) plane for which the two types of calculations lead to similar GSF profiles, solution of the PN equation gives comparable extension of the screw dislocation core. For the potential model we find a dislocation core half-width of $1.4 \AA$, close to the value of $1.9 \AA$ found by Durinck et al. [7].

The geometry of the core of the [001] screw dislocation as predicted by direct calculation is presented in Figure 2. The $\mathrm{SiO}_{4}$ tetrahedra are preserved in the dislocation core. Few atomic positions differ from those in the perfect crystal since the dislocation line is viewed end-on. This emphasises the fact that atomic relaxation in the core are mostly parallel to the Burgers vector. To vizualize the dislocation core geometry, the differential displacement of the oxygen sublattice is superimposed on the core structure in Figure 2. This clearly shows that the core is spread in several planes. This is dramatically different to the displacement predicted by the Peierls dislocation, which, as expected and shown in Figure 3, is confined within a single plane.

\section{Discussion}

Whatever model is chosen, Figures 2 and 3 clearly show that the maximum relative displacements occur between atoms that do not belong to the same tetrahedra. This confirms that $\mathrm{Si}-\mathrm{O}$ bonds are stiff and the tetrahedra act like rigid units. The total dislocation density is spread in such a way that the $\mathrm{SiO}_{4}$ tetrahedra are not deformed. The Peierls dislocation shown in Figure 3 has been constructed assuming a planar core. Indeed, the arrows are observed within the (010) layer located between two layers of tetrahedra. Compared to Figure 3, the dislocation shown in Figure 2 clearly deviates from a planar core since arrows are found in several layers. A large part of the dislocation density profile is located at the centre, between 
the tetrahedra. A significant part of shear is spread out in two (010) planes. As a result, the [001] dislocation clearly exhibits a 3D core structure as anticipated by Durinck et al. [8]. The strong tendency of the shear distribution to be located in two (010) planes is very similar to the so-called zonal dissociation proposed for $<\mathrm{a}>$ dislocations in quartz [47].

We now have to ask what is physical relevance of these two models for the dislocation core structure? To answer this question, one must remember how they have been constructed. The Peierls model imposes a planar core. Hence, this dislocation is, by construction, the most able to glide in a given plane. No constraint is put on its energy. On the contrary, the direct model derives from an energy minimisation process. This dislocation is likely to be the most "stable" (lowest energy configuration), but not necessarily the most easy to glide. Indeed, we calculated the energy stored within the inner $10 \AA$ of both models embedded within a $30 \AA$ representation of the extended dislocated crystal while taking care to include the same atoms in each setup. This calculation showed that the PN core is up to $2.7 \mathrm{eV} / \AA$ higher in energy than the fully relaxed configuration (this is an upper bound on the energy difference as the location of the oxygen shells in the PN model can not be determined and the oxygen is thus not polarized).

We now discuss the implications of those two possible core configurations on dislocation mobility. Vitek suggested that the kink-pair mechanism in bcc metals corresponds to the transformation of a non-planar core into a planar core in the slip plane [48]. At rest, [001] dislocations in olivine are likely to exhibit the stable - non-planar - sessile configuration of Figure 2. This is consistent with the observation of long [001] screw dislocations. One can suppose that, under stress and in order to move, the core would need to transform into the metastable - planar - glissile configuration described by the Peierls model (Figure 3). Molecular dynamics modelling, perhaps along the lines reported by Marian et al. [49] for bcc iron, appears to be the most promising technique to probe this process. However, the important parameter is clearly the energy difference between the two cores, which is found to be large in the present case: $2.7 \mathrm{eV} / \AA$. To give an idea, this energy difference is significantly larger than the highest Peierls energy barrier for glide of [100] Peierls dislocations (1.9 eV/ $\AA$ ), which requires a stress of almost $30 \mathrm{GPa}$ to move [8]. Of course, core transformation processes will also be thermally activated. The energy difference for the sessile-glissile transitions must also be compared with the Peierls energy responsible for the friction opposed to glide of the glissile core. For [001](010) screw dislocations, the Peierls energy is $0.13 \mathrm{eV} / \AA$ [8], a value significantly lower that the threshold energy. The stress necessary to move the glissile dislocation is thus much lower than the threshold stress required to transform the core 
into the glissile configuration. This is characteristic of an unlocking mechanism as described by Couret and Caillard [50]. In that case, the metastable dislocations glide rapidly over a freeglide distance before falling back into a stable configuration (locking). The average mobility corresponding to this jerky motion is controlled by the unlocking and locking events. In situ deformation experiments in a transmission electron microscope would be highly desirable to check if this mechanism operates.

\section{Conclusion}

The geometrical analysis of differential displacements within the core of a [001] screw dislocation suggests a 3D core as suggested by Durinck et al [8]. This observation is consistent with the microstructures observed in the TEM, where long screw segments are observed. Further modelling of the mobility of [001] dislocations is needed for a better understanding of the dislocation core under stress, of its ability to glide and possibly of the ability of the core to switch from a sessile (non-planar) configuration to a more mobile (planar) structure.

Acknowledgements: The authors warmly thank Dr. R. Groger for his help and for allowing use of the ddplot program. AMW acknowledges funding under the NERC Postdoctoral Research Fellowship scheme (NE/E012922/1). 


\section{References}

[1] D.L. Kohlstedt and C. Goetze, J. Geophys. Res. 79 (1974) p.2045.

[2] W.B. Durham, C. Goetze and B. Blake, J. Geophys. Res. 82 (1977) p.5755.

[3] Y. Gueguen and M. Darot, Philos. Mag. A 45 (1982) p.419.

[4] P. Cordier, in Plastic deformation of minerals and rocks, edited by S.-i. Karato and H.R. Wenk (Mineral Society of America, Washington, DC, 2002), Vol. 51.

[5] R. Von Mises, Z. Angew. Math. Mech. 8 (1928) p.161.

[6] H. Couvy, D. Frost, F. Heidelbach, K. Nyilas, T. Ungár, S. Mackwell and P. Cordier, Eur. J. Mineral. 16 (2004) p.877.

[7] J. Durinck, A. Legris and P. Cordier, Am. Mineral. 90 (2005) p.1072.

[8] J. Durinck, P. Carrez and P. Cordier, Eur. J. Mineral. 19 (2007) p.631.

[9] D. Mainprice, A. Tommasi, H. Couvy, P. Cordier and D.J. Frost, Nature 433 (2005) p.731.

[10] P. Raterron, J.H. Chen, L. Li, D.J. Weidner and P. Cordier, Am. Mineral. 92 (2007) p.1436.

[11] H.Y. Jung and S. Karato, Science 293 (2001) p.1460.

[12] H. Jung, I. Katayama, Z. Jiang, I. Hiraga and S. Karato, Tectonophysics 421 (2006) p.1.

[13] M.S. Duesbery and V. Vitek, Acta Mater. 46 (1998) p.1481.

[14] G. Wang, A. Strachan, T. Cagin and W.A. Goddard III, Phys. Rev. B 68 (2003) p.224101.

[15] J. Chaussidon, M. Fivel and D. Rodney, Acta Mater. 54 (2006) p.3407.

[16] P. Phakey, G. Dollinger and J.M. Christie, Geophys Monogr. Ser. - Flow and Fracture of Rocks 13 (1972) p.117.

[17] A.M. Walker, J.D. Gale, B. Slater and K. Wright, Phys. Chem. Chem. Phys. 7 (2005) p.3227.

[18] A.M. Walker, J.D. Gale, B. Slater and K. Wright, Phys. Chem. Chem. Phys. 7 (2005) p.3235.

[19] G. Schoeck, Mater. Sci. Eng. A 400-401 (2005) p.7.

[20] G. Schoeck, Acta Materialia 54 (2006) p.4865.

[21] C. Woodward, Mater. Sci. Eng. A 400-401 (2005) p.59.

[22] C. Woodward, D.R. Trinkle, L.G.J. Hector and D.L. Olmsted, Phys. Rev. Lett. 100 (2008) $\operatorname{art.n}^{\circ} 045507$. 
[23] R. Schroll, V. Vitek and P. Gumbsch, Acta Mater. 46 (1998) p.903.

[24] G. Schoeck, Acta Mater. 49 (2001) p.1179.

[25] C.R.A. Catlow, Proc. R. Soc. Lond. Ser. A Math. Phys. Eng. Sci. 353 (1977) p.533.

[26] M.J. Sanders, M. Leslie and C.R.A. Catlow, Journal of the Chemical Society, Chemical Communications (1984) p.1271.

[27] G.V. Lewis and C.R.A. Catlow, J. Phys. C 18 (1985) p.1149.

[28] G.D. Price, S.C. Parker and M. Leslie, Phys. Chem. Minerals 15 (1987) p.181.

[29] G.D. Price, S.C. Parker and M. Leslie, Min. Mag. 51 (1987) p.157.

[30] S.C. Parker and G.D. Price, Adv. Solid-State Chem. 1 (1989) p.295.

[31] G.W. Watson, P.M. Oliver and S.C. Parker, Phys. Chem. Minerals 25 (1997) p.70.

[32] N.H. de Leeuw, S.C. Parker, C.R.A. Catlow and G.D. Price, Phys. Chem. Minerals 27 (2000) p.332.

[33] N.H. de Leeuw, S.C. Parker, C.R.A. Catlow and G.D. Price, Am. Mineral. 85 (2000) p.1143.

[34] K. Wright and C.R.A. Catlow, Phys. Chem. Minerals 20 (1994) p.515.

[35] J.A. Purton, N.L. Allan, J.D. Blundy and E.A. Wasserman, Geochim. Cosmochim. Acta 60 (1996) p.4977.

[36] J.A. Purton, N.L. Allan and J.D. Blundy, Geochim. Cosmochim. Acta 61 (1997) p.3927.

[37] A.M. Walker, S.M. Woodley, B. Slater and K. Wright, Phys. Earth Planet. Int. in press (2008).

[38] V.R. Saunders, C. Freyria-Fava, R. Dovesi and C. Roetti, Comput. Phys. Comm. 84 (1994) p.156.

[39] J.D. Gale and A.L. Rohl, Mol. Simulat. 29 (2003) p.291.

[40] B. Joos, Q. Ren and M.S. Duesbery, Phys. Rev. B 50 (1994) p.5890.

[41] J.D. Eshelby, Philos. Mag. A 40 (1949) p.903.

[42] J.P. Hirth and J. Lothe, Theory of dislocations, John Wiley \& Sons, Inc, New York, 1982.

[43] J.F. Nye, Physical properties of crystals, Oxford University Press, Oxford, UK, 1957.

[44] J.W. Steeds, Introduction to Anisotropic Elasticity Theory of Dislocations, Clarendon Press, Oxford, UK, 1973.

[45] V. Vitek, R.C. Perrin and D.K. Bowen, Philos. Mag. A 21 (1970) p.1049.

[46] A.M. Walker, K. Wright and B. Slater, Phys. Chem. Minerals 30 (2003) p.536.

[47] L. Trépied and J.C. Doukhan, Phys. Stat. Sol (a) 49 (1978) p.713. 
[48] V. Vitek, Phys. Stat. Sol (b) 18 (1966) p.687.

[49] J. Marian, W. Cai and V.V. Bulatov, Nature Materials 3 (2004) p.158.

[50] A. Couret and D. Caillard, Philos. Mag. A 59 (1989) p.783. 


\begin{tabular}{|c|c|c|c|}
\hline \multicolumn{3}{|c|}{ Charges (units of electronic charge) } & \multirow{2}{*}{$\begin{array}{l}\text { Core }- \text { shell spring } \\
\text { constant }\left(e V \AA^{-2}\right)\end{array}$} \\
\hline Ion & Core & Shell & \\
\hline $\mathrm{Mg}$ & 2.0 & & \\
\hline $\mathrm{Si}$ & 4.0 & & \\
\hline $\mathrm{O}$ & 0.84819 & -2.84819 & 74.92038 \\
\hline $\begin{array}{l}\text { Buckingham } \\
\text { potential }\end{array}$ & $A(\mathrm{eV})$ & $\rho(\AA)$ & $C\left(\mathrm{eV} \AA^{-6}\right)$ \\
\hline $\mathrm{Si}-\mathrm{O}$ & 1283.90734 & 0.32052 & 10.66158 \\
\hline $\mathrm{Mg}-\mathrm{O}$ & 1428.5 & 0.29453 & 0.0 \\
\hline $\mathrm{O}-\mathrm{O}$ & 22764.0 & 0.1490 & 27.88 \\
\hline Harmonic three-body & $k\left(\mathrm{eVrad}^{-2}\right)$ & $\theta_{0}$ (degrees) & \\
\hline $\mathrm{O}-\mathrm{Si}-\mathrm{O}$ & 2.09724 & 109.47 & \\
\hline
\end{tabular}

Table 1: Parameters of the potential model. Pair potentials are truncated beyond $10 \AA$ and three body terms only act between bonded $\mathrm{SiO}_{4}$ groups.

\begin{tabular}{|c|c|c|c|c|c|c|}
\hline & \multicolumn{3}{|c|}{ DFT } & \multicolumn{3}{c|}{ Parameterised potentials } \\
\hline & $\begin{array}{c}\gamma^{\max } \\
\left(\mathrm{eV} / \AA^{2}\right)\end{array}$ & $\begin{array}{c}\text { SFE } \\
\left(\mathrm{eV} / \AA^{2}\right)\end{array}$ & $\gamma^{\max } / \mathrm{SFE}$ & $\begin{array}{c}\gamma^{\max } \\
\left(\mathrm{eV} / \AA^{2}\right)\end{array}$ & $\begin{array}{c}\text { SFE } \\
\left(\mathrm{eV} / \AA^{2}\right)\end{array}$ & $\gamma^{\max } / \mathrm{SFE}$ \\
\hline$\{110\}$ & 0.077 & 0.024 & 3.2 & 0.115 & 0.042 & 2.7 \\
\hline$(100)$ & 0.037 & 0.019 & 1.9 & 0.048 & 0.043 & 1.1 \\
\hline$(010)$ & 0.128 & - & - & 0.150 & - & - \\
\hline
\end{tabular}

Table 2: Principal parameters, $\gamma^{\max }$ and stacking fault energy (SFE), of GSF calculations depending on the calculation method. 


\section{Figures}

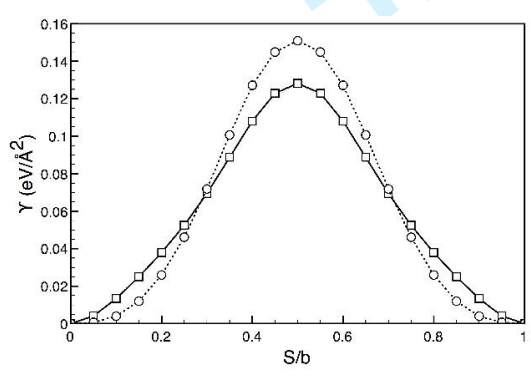

(a)

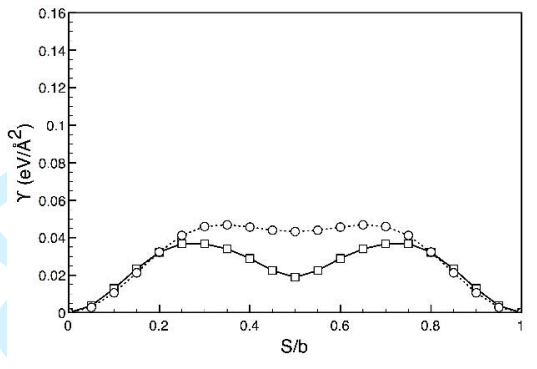

(b)

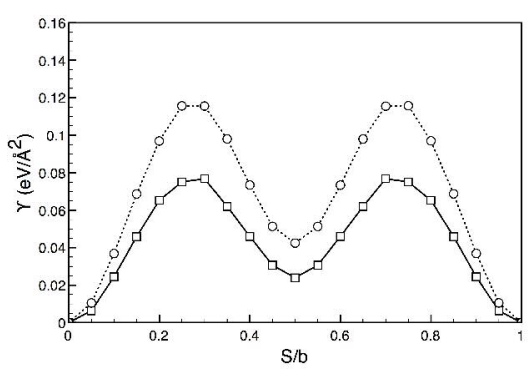

(c)

Figure 1: Generalised Stacking Faults (GSF) corresponding to a [001] shear in (010), (100) and (110). The GSF are given for both VASP (squares) and GULP (circles) calculations as a function of $\mathrm{S} / \mathrm{b}$ where $\mathrm{S}$ is the shear amount and $\mathrm{b}$ the modulus of [001]. 


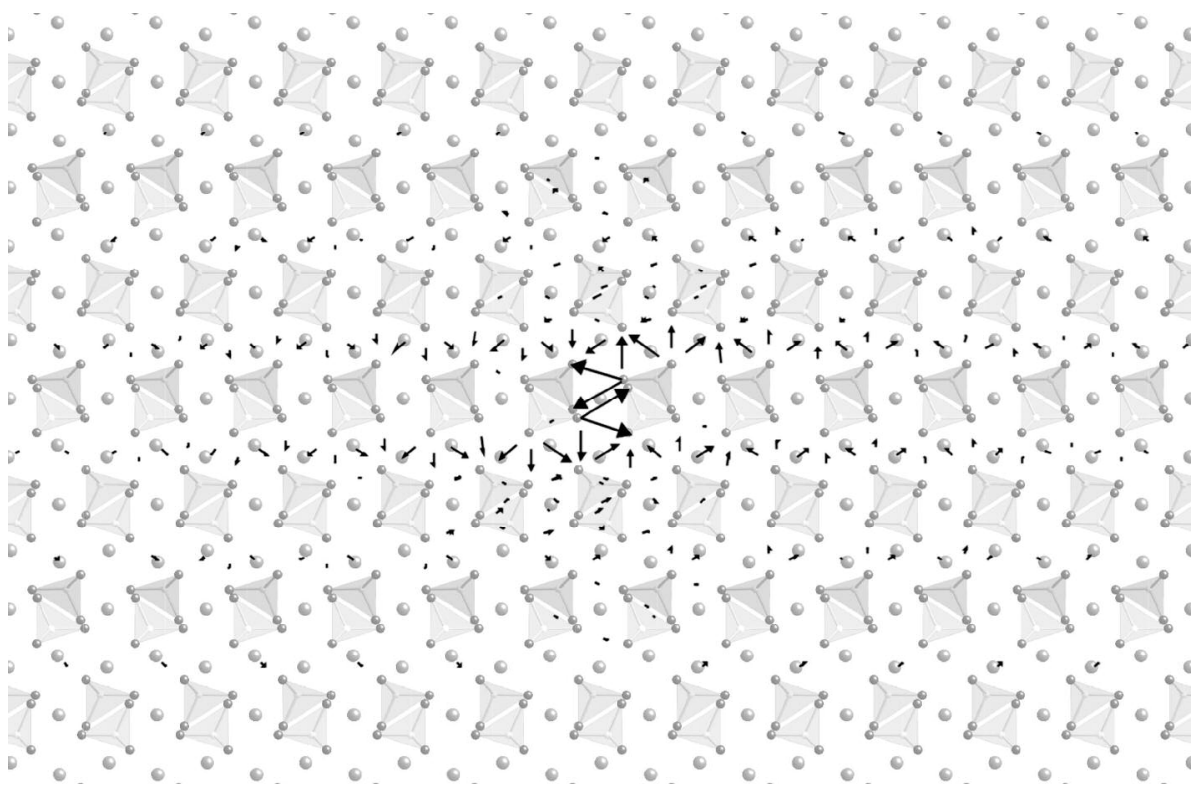

Figure 2: Differential displacement map for the [001] screw dislocation calculated by direct simulation by Walker et al. [18]. The dislocation is viewed end on (i.e. along the [001] direction)

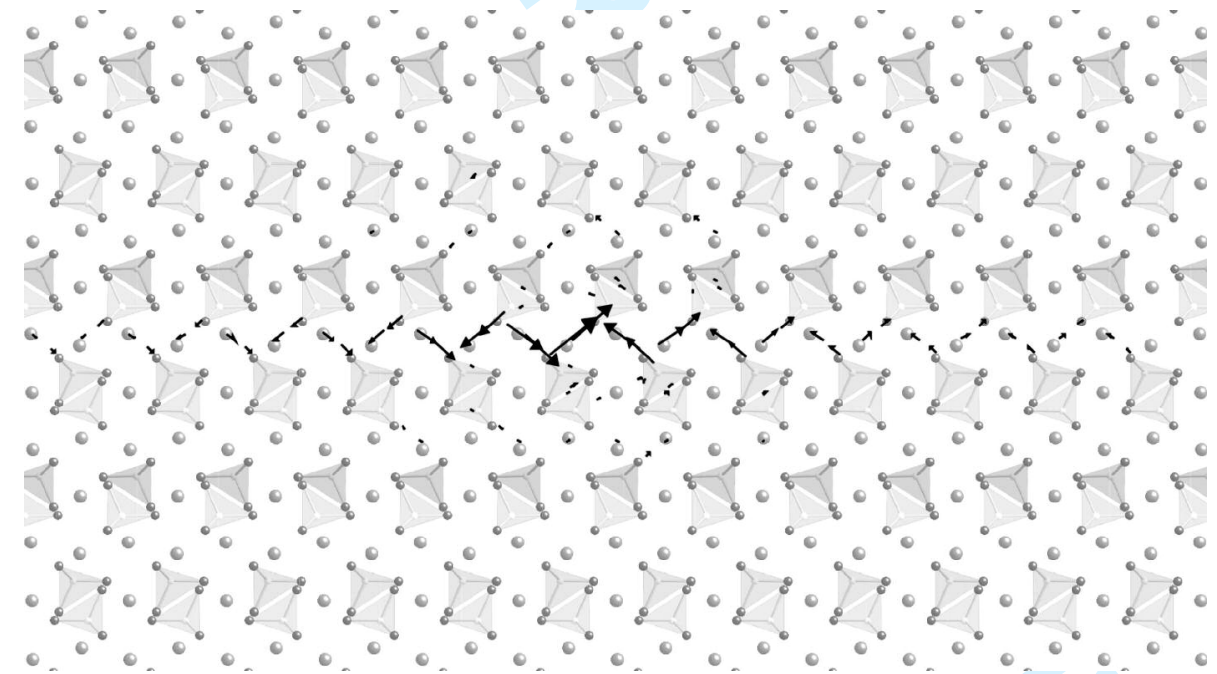

Figure 3: Differential displacement map for the Peierls [001](010) screw dislocation 


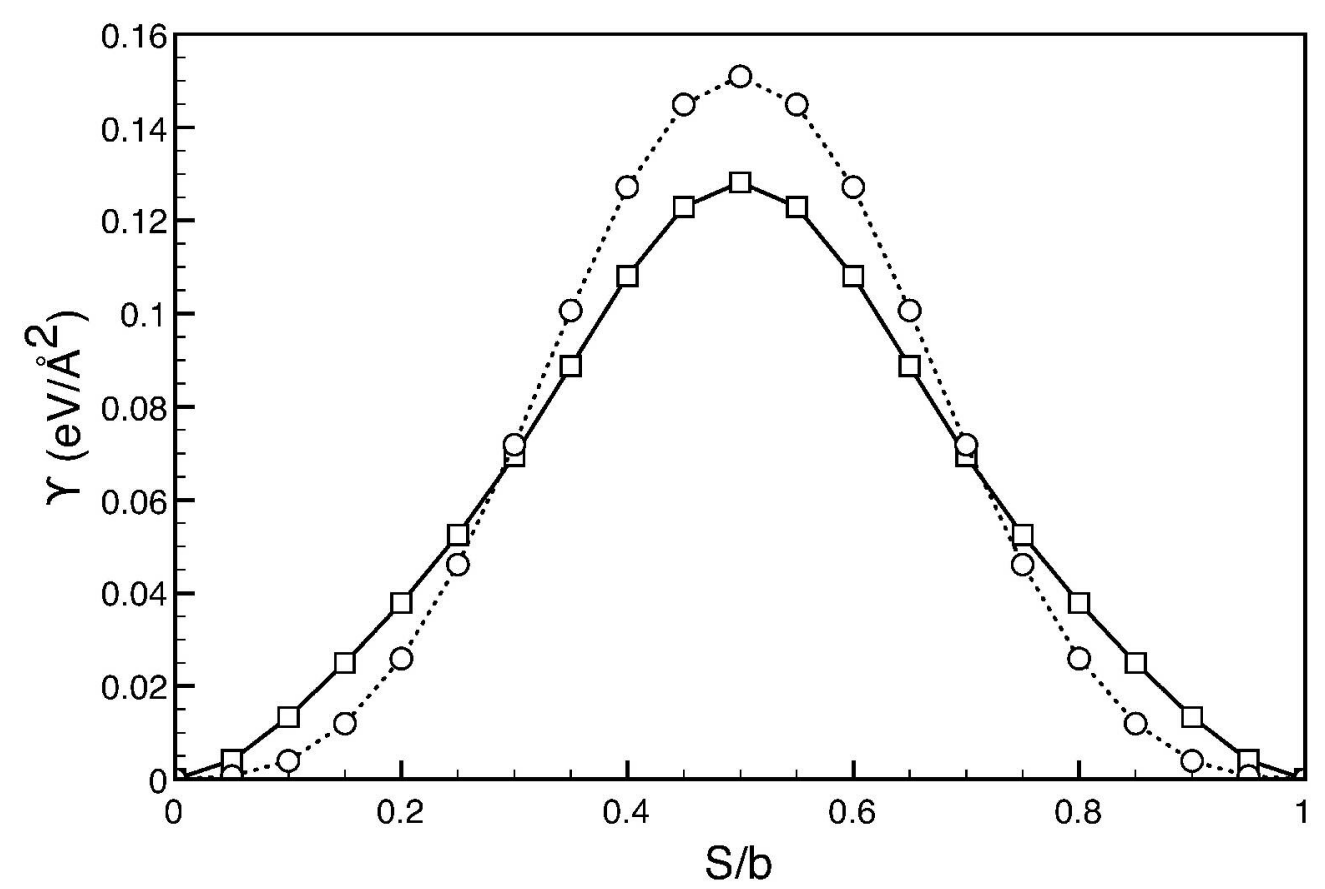

$239 \times 169 \mathrm{~mm}(600 \times 600 \mathrm{DPI})$ 


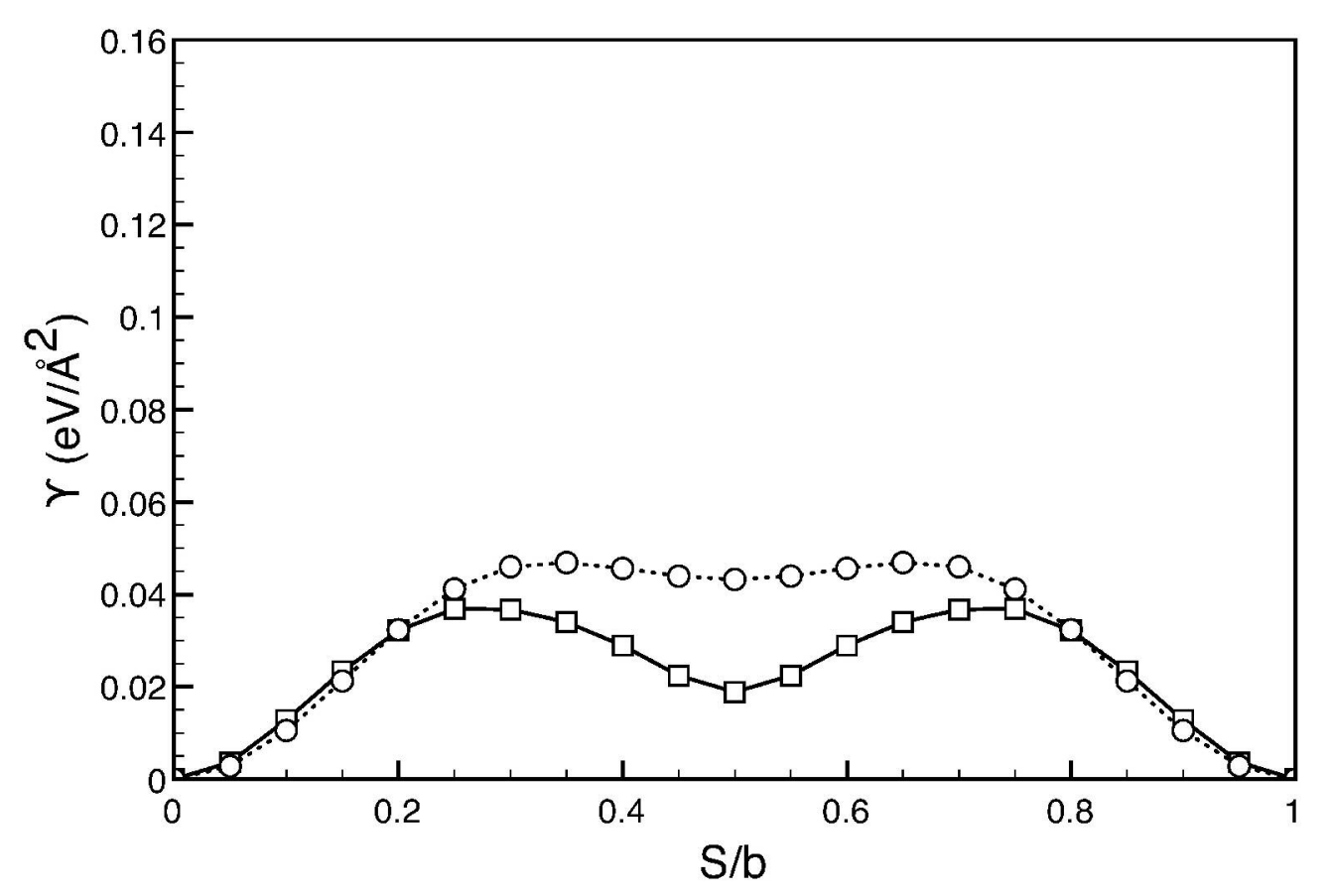

$239 \times 169 \mathrm{~mm}(600 \times 600 \mathrm{DPI})$ 


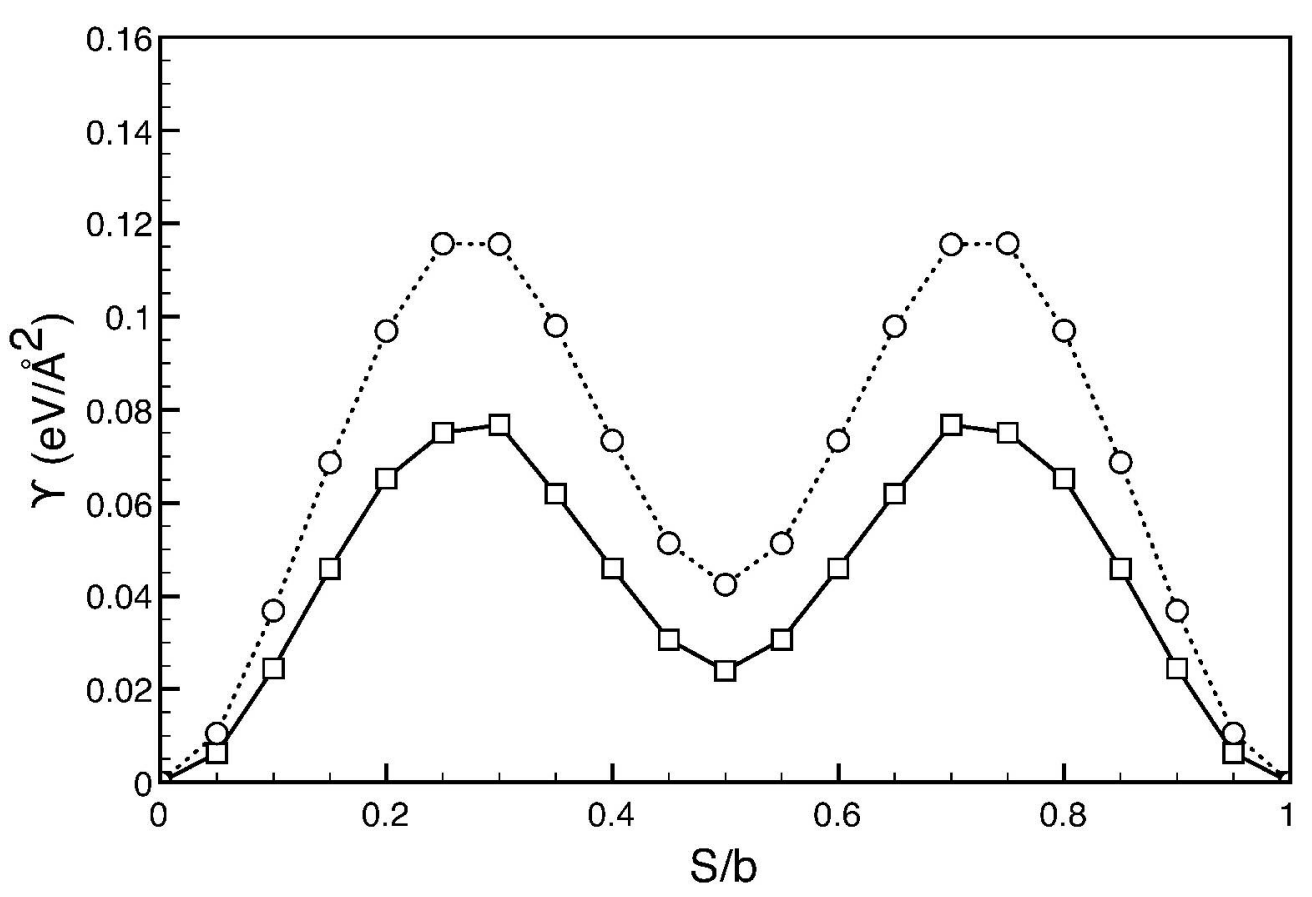

$239 \times 169 m m(600 \times 600$ DPI $)$ 


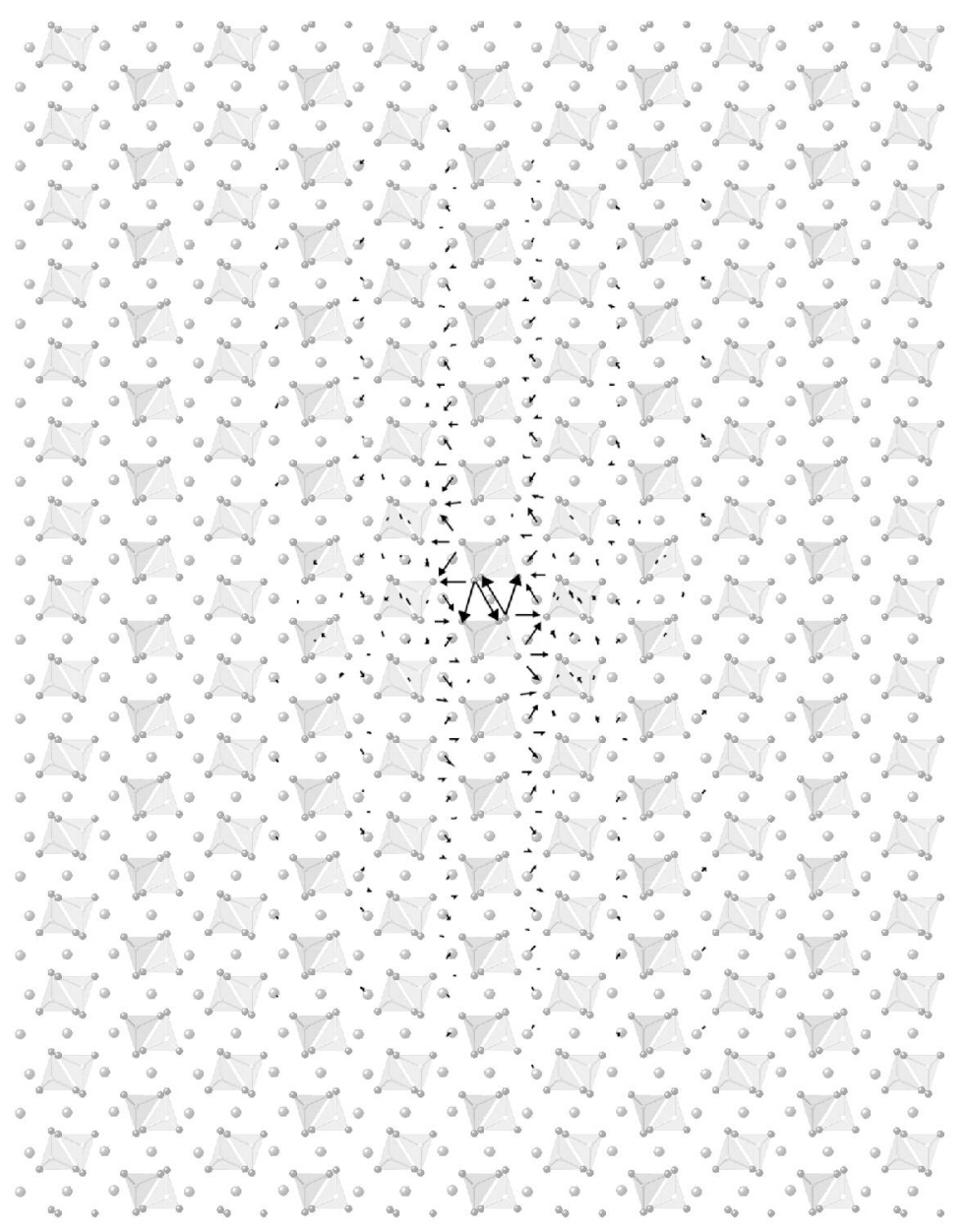

$209 \times 297 \mathrm{~mm}(600 \times 600 \mathrm{DPI})$ 


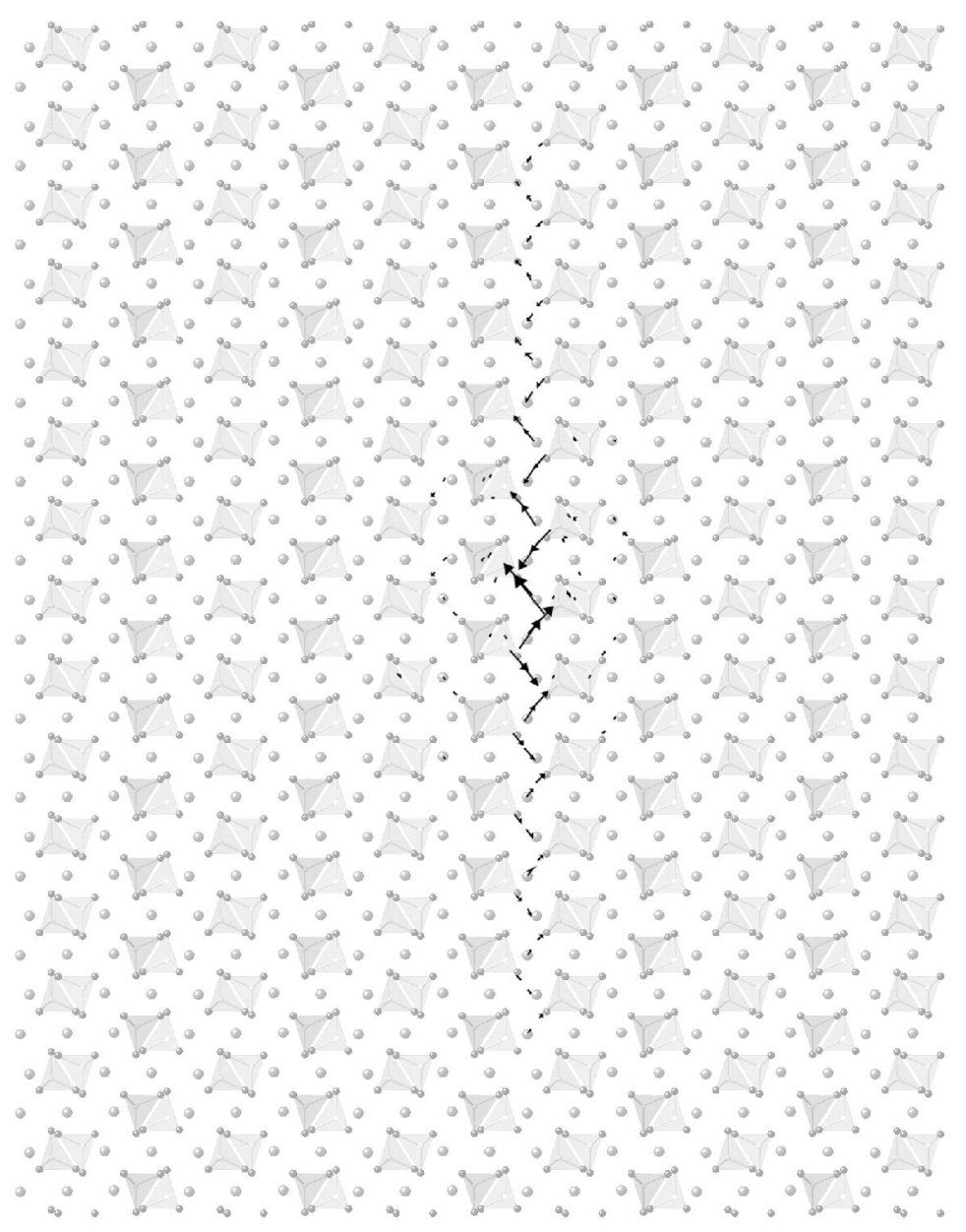

$209 \times 297 \mathrm{~mm}(600 \times 600$ DPI $)$ 